\title{
Comprehending compass directions
}

\author{
GEOFFREY R. LOFTUS \\ University of Washington, Seattle, Washington 98195
}

\begin{abstract}
Two experiments investigated the processes by which compass directions are comprehended. In both experiments, the task was as follows. A compass direction (e.g., $210 \mathrm{deg}$ ) was visually presented. The subject drew a representation of the direction and then pressed a key. Reaction time from onset of the stimulus to the keypress was measured. The results suggested a model of direction comprehension involving two steps: first, computing the nearest cardinal direction to the target direction, and then "rotating" from the cardinal to the target direction. Rotation could be performed equally well clockwise or counterclockwise. Additionally, north-south tended to be comprehended faster than east-west, confirming past results that have shown an advantage of up-down over left-right in discrimination tasks.
\end{abstract}

Most creatures are perpetually faced with the task of figuring out where they are going and how they are going to get there. Consequently, the question of how these feats are accomplished is of some interest to psychologists. A seminal paper by Tolman (1948) introduced the concept of a "cognitive map," and there appears to be ample evidence that some internal representation of the environment plays a major role in guiding locomotive behavior, both for animals (as Tolman and his co-workers demonstrated so elegantly) and for humans (Downs \& Stea, 1973; Lynch, 1960).

Many humans possess a convenient aid to finding their way around, namely, a system of compass directions, such as north, south, east, and west. Since compass directions are fixed with respect to the environment within which one is attempting to navigate (the earth), they provide directional information that is invariant over a wide range of circumstances (e.g., invariant with respect to one's particular, momentary orientation, one's geographical location, and so on). As such, the system of compass directions constitutes a fundamental tool by which locational information is transmitted from person to person, and possibly also a fundamental structure within which one constructs an internal representation of one's geographical surround.

The present experiments are exploratory in nature and address three interrelated issues concerning representation of and utilization of directional information by humans. The first issue involves the

This study was supported by National Science Foundation Grant BNS75-10450 to the author. Portions of the data from Experiment 1 were presented at an ARPA conference on cartography, San Francisco, 1976. Thanks go to Beth MacGregor for her assistance in running subjects for Experiment 2 and to Veronica Dark who wrote the computer program to run the experiments, assisted in running subjects, and performed all data analyses. I also thank Louise Carter-Saltzman, Buz Hunt, Colin MacLeod, and Tom Nelson for their comments on the manuscript. Requests for reprints may be sent to Geoffrey $\mathbf{R}$. Loftus, Department of Psychology, University of Washington, Seattle, Washington 98195. processes by which external information corresponding to a particular direction is comprehended and utilized. The second issue concerns the extent to which such processes may be modified by changing the way in which the to-be-acted-on information is transmitted. The third issue involves the manner in which directional information is permanently represented. To investigate these issues, a paradigm was devised that consisted of the following. On each of a series of trials, a subject was (visually) presented with a compass direction between 0 and $350 \mathrm{deg}$. The subject's task was to indicate comprehension of the direction by drawing a representation of it and then pushing a key. The reaction time (RT) between the onset of the stimulus and the keypress was then used as an indicant of the time to comprehend the direction. Functions relating RT to (1) the specific direction presented and (2) the way in which the directional information was transmitted can then be used to make inferences about the manner in which compass directions are represented and processed.

\section{EXPERIMENT 1}

The original motivation for carrying out Experiment 1 was primarily a practical one and involved the following considerations. In most current navigation and communication systems (e.g., the Air Traffic Control system), a compass direction is transmitted in the form of a single number ranging from 0 to $359 \mathrm{deg}$ (with 0 representing north, 90 representing east, 180 representing south, and 270 representing west). An informal survey of airplane pilots revealed some strong intuitions about how some particular direction (say $210 \mathrm{deg}$ ) is comprehended. In particular, two major steps seemed to be involved. First, the nearest cardinal direction (i.e., the nearest multiple of 90 ; in the present example, south, or $180 \mathrm{deg}$ ) is computed, and one "mentally faces" in that direction. Second, the difference between the cardinal direction and the desired direction is computed and a "mental rotation" (either 
clockwise or counterclockwise) is carried out until the desired direction is reached.

Experiment 1 was designed (1) to investigate the viability of these introspections and (2) to see whether the first step (computing the nearest cardinal direction) could be short-circuited by providing an appropriate cardinal direction as part of the transmitted information.

\section{Method}

Subjects. Two separate groups of subjects participated in Experiment 1. The first group consisted of eight University of Washington undergraduates who responded to an advertisement in the campus newspaper and who were relatively unpracticed in the use of directional information. The second group consisted of three Navy pilots, each of whom had more than $3,000 \mathrm{~h}$ of flying time and was highly practiced with respect to comprehension and utilization of directional information. All subjects were paid $\$ 2$ for participating.

Apparatus. Stimulus presentation and response collection were performed by an on-line NOVA 820 computer. Stimul were displayed on Tektronics cathode-ray tubes (CRTs) and the principal response consisted of pressing a key on a custom-built keyboard. Up to four subjects were run at a time, each in an individual booth, and each at his or her own rate.

Stimuli. Stimuli consisted of information corresponding to the compass directions from 0 to $350 \mathrm{deg}$ in increments of $10 \mathrm{deg}$ (producing a total of 36 separate directions). The information corresponding to a particular direction was presented in one of two ways. The numerical method is the standard method currently used for presenting directional information. Very simply, a given direction consists of a single number representing degrees of rotation, clockwise from north and ranging from 0 to 350 . The second method was dubbed the quadrant method. In the quadrant method, the compass rose is viewed as being divided into four quadrants: The north quadrant includes all directions from 0 to $89 \mathrm{deg}$, the east quadrant includes directions from 90 to $179 \mathrm{deg}$, and so on. A particular direction is then transmitted as a cardinal direction plus some number ranging from 0 to 80 representing position within the corresponding quadrant. So, for example, $210 \mathrm{deg}$ would, in the quadrant method, be represented as "south plus 30."

Design and Procedure. Each subject participated in a single experimental session, lasting about $1 \mathrm{~h}$. The session was divided into blocks: three blocks for the undergraduates and four blocks for the pilots. A block, in turn, consisted of 72 trials made up of one presentation apiece of each of the 36 directions in each of the two possible presentation methods. Order of the 72 separate conditions was randomized over the 72 trials There was a short break after the 36th trial of each block.

Each subject had a clipboard, affixed to which was a sheet of paper containing 36 circles, each circle approximately $3 \mathrm{~cm}$ in diameter. Each circle represented a compass rose and had (1) a dot in the center and (2) a number beside it ranging from 1 to 36. Each circle was to be used for one particular trial. Subjects held a pencil with the preferred hand, poised over the clipboard.

On each trial, the following series of events occurred. (1) The message "Center pencil in Circle $n$ " (where $n$ was the trial number within a half block) appeared at the center of the screen. The subject then placed his or her pencil at the center of the appropriate circle. (2) The message disappeared and, following a .5 -sec interval, a direction was presented where the message had just been. (3) The subject responded by drawing a line from the center of the circle to the perimeter in the direction signaled by the stimulus. Subjects were instructed to respond as quickly as possible and to be accurate within \pm 10 deg. (4) After drawing the line, the subject pressed a key with his or her nonpreferred hand. The keypress effected the disappearance of the stimulus and ended the trial. A 2-sec intertrial interval then followed.

Subjects were able to carry out the sequence of responses (drawing the line and then immediately pressing the key) with surprisingly little difficulty. As a precaution against gross cheating (pressing the key and then drawing the line), an error message appeared if any response was made in less than $1 \mathrm{sec}$.

Between each block and after the 36th trial in each bilock, the paper on the clipboard was replaced. At the start of the session, subjects were given instructions about compass directions, the two presentation methods, and the experimental procedure. Following instructions and questions, subjects were provided with 36 practice trials.

\section{Results}

A representation was deemed to be correct if it was within $\pm 10 \mathrm{deg}$ of the correct orientation. By this (somewhat arbitrary) criterion, virtually all responses were correct. Analyses exclude the few errors that were made.

For each subject, a median reaction time (RT) was computed for each of the 72 conditions. Means of these medians were then computed across subjects.

For purposes of analysis, the 72 conditions were characterized as resulting from three factorially combined variables: 4 quadrants by 9 positions $(0-80 \mathrm{deg})$. within a quadrant by 2 presentation methods. A repeatedmeasures analysis of variance (ANOVA) showed no significant ${ }^{1}$ Quadrant by Position interaction either for naive subjects $[\mathrm{F}(24,168)=1.05, \mathrm{MSe}=12.39]^{2}$ or for pilots $[\mathrm{F}(24,48)=1.24, \quad \mathrm{MSe}=5.25]$. Additionally, the three-way interactions were nonsignificant (both Fs $<1$ ). Therefore, the data are shown collapsed across each of these factors in turn.

Position within a quadrant. Figure 1 shows RT as a function of position within a quadrant for each of the two presentation methods. Data are shown separately for undergraduates and for pilots, as it seemed reasonable to expect that the pilots' far greater experience with directional information might lead to a different pattern of results from that of the undergraduates. As it turns out, however, although the pilots were faster than the undergraduates (by about
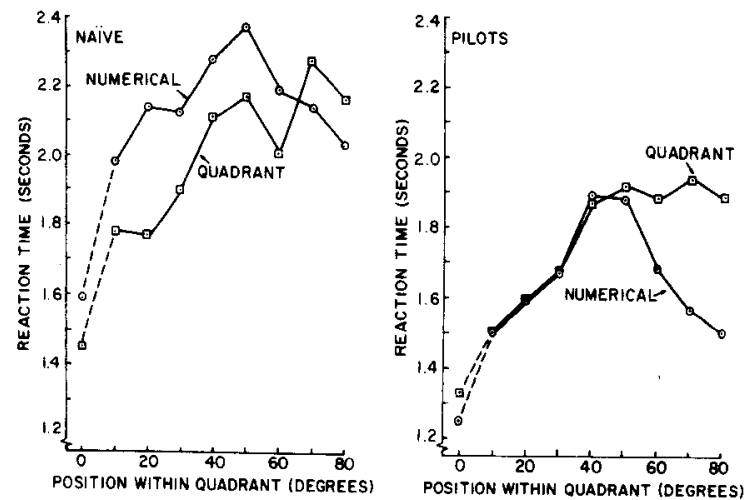

Figure 1. Experiment 1: Reaction time as a function of position within a quadrant. 
$500 \mathrm{msec}$ ), the two patterns of results were remarkably similar. The effect of presentation method was not statistically significant for either group $[F(1,7)=2.37$, $\mathrm{MSe}=110.20$ for naive subjects and $F(1,2)=2.46$, $\mathrm{MSe}=30.36$ for pilots]. However, as the data in Figure 1 suggest, there were strong effects due to position $[\mathrm{F}(8,56)=17.40, \quad \mathrm{MSe}=18.60$ for naive subjects and $F(8,16)=24.03, \mathrm{MSe}=3.70$ for pilots] and to the Position by Presentation Method interaction $[F(8,56)=3.09$, MSe $=13.87$ for naive subjects and $\mathrm{F}(8,16)=6.43, \mathrm{MSe}=2.31$ for pilots $]$. When the numerical method was used, the function relating RT to position was nonmonotonic, rising to a peak at $40.50 \mathrm{deg}$ and then falling again. When the quadrant method was used, on the other hand, the RT function rose through 40-50 deg and then flattened out.

Different quadrants. Figure 2 shows the functions relating $\mathrm{RT}$ to the particular quadrant being tested. Again, although quantitative differences existed between pilots and undergraduates, the patterns of results were exceedingly similar. There were strong effects due to quadrant $[\mathrm{F}(3,21)=21.28, \mathrm{MSe}=18.60$ for naive subjects and $F(3,6)=27.28, \mathrm{MSe}=2.38$ for pilots] and to the Quadrant by Presentation Method interaction $[F(3,21)=7.99, \mathrm{MSe}=30.34$ for naive subjects and $\mathrm{F}(3,6)=19.24, \mathrm{MSe}=1.03$ for pilots]. When the numerical method was used, RT increased monotonically from north, around the compass rose, to west. Quite a different pattern of results emerged when the quadrant method was used. Here, RT was relatively short for the north and south quadrants, but about $250 \mathrm{msec}$ longer for the east and west quadrants.

\section{Discussion}

The results of Experiment 1 must be viewed as preliminary and exploratory, but they do provide some suggestions bearing on what subjects do and do not do when going about comprehending directional information (at least in the present task).
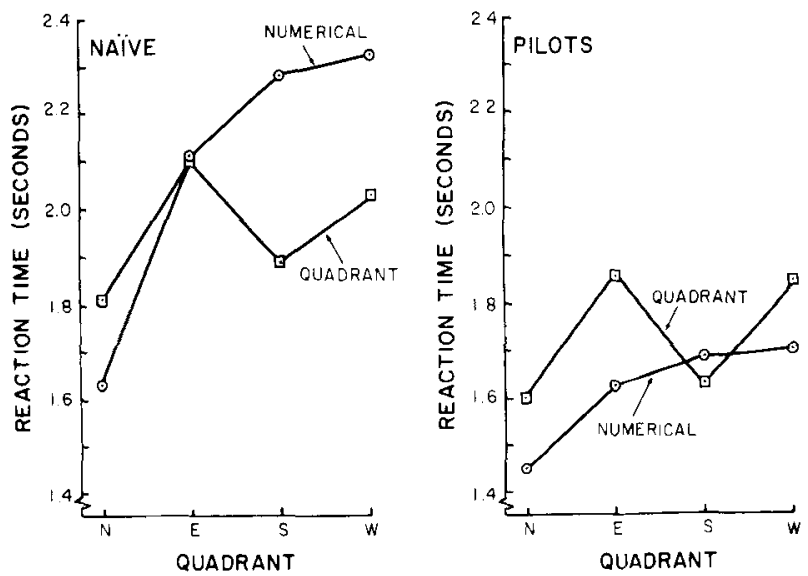

Figure 2. Experiment 1: Reaction time for the four quadrants.
The fact that different patterns of results emerge from the different stimulus presentation methods rules out any model of direction comprehension positing that the only determinant of how quickly directional information is understood is the actual direction to which the information corresponds. Examples of such models would include the following: (1) Any compass direction has associated with it some degree of familiarity (perhaps due to its frequency of use) and time to understand the direction is determined solely by its frequency. (2) In the particular paradigm used in this experiment, variation in reaction time merely reflects the relative ease of drawing a line in some particular orientation.

Mental operations involved in comprehension of direction. Rather, the results of Experiment 1 imply that a direction is understood via a series of mental operations and that the particular operations involved, as well as the order in which they are carried out, is dependent on the form of the information that initiates them. A first approximation to a model of direction comprehension could reasonably reflect the introspections described earlier; that is, it would involve two major processes: (1) Some appropriate cardinal direction is identified and then (2) some operation is undertaken that is akin to a rotation from the cardinal direction to the desired direction. (The term "rotation" is used here only descriptively, to reflect the fact that the transition time from one direction to another is an increasing function of the angular distance between the two directions). The form of the numerical method data in Figure 1 suggest (1) that in the absence of a given cardinal direction, the cardinal direction that is selected is the one nearest to the presented direction and (2) that counterclockwise rotation is performed as fast as clockwise rotation. The basis of the latter assertion is that the RT functions for the numerical presentation method are almost perfectly symmetrical around $45 \mathrm{deg}$.

\section{EXPERIMENT 2}

Experiment 2 was carried out for several reasons. First, it seemed worthwhile simply to ascertain that the data of Experiment 1 would replicate. Second, the randomization procedure of Experiment 1 resulted in subjects' not knowing the method by which information would be presented on any given trial. This in turn meant that the extra processes of evaluating the presentation method and perhaps formulating a strategy had to be carried out on each trial. Since it was not known how the necessity of carrying out these extra steps would interact with other processes, a condition was introduced in Experiment 2, whereby for some subjects trials were blocked by presentation method. Third, subjects in Experiment 2 were given a good deal more practice than were subjects in Experiment 1: 
Each subject participated in three experimental sessions rather than only one. Finally, in Experiment 2 there was introduced a new presentation method consisting of a cardinal direction minus some number between 0 and $80 \mathrm{deg}$. Hence, Experiment 2 had three presentation methods that are dubbed "numerical" (e.g., "210"), "quadrant-add" (e.g., "south plus 30"), and "quadrant-subtract" (e.g., "west minus 60"). The "quadrant-subtract" presentation method was concocted to test the notion that rotation to some desired direction can take place equally well clockwise or counterclockwise from a cardinal direction. If such is the case then the within-quadrant RT functions for the quadrant-plus and quadrant-minus conditions should be mirror images of each other.

\section{Method}

Subjects. Subjects were 16 University of Washington undergraduates who responded to an advertisement in a campus newspaper and were paid $\$ 2 / \mathrm{h}$. All subjects were relatively unpracticed with respect to the use of directional information.

Apparatus. The apparatus was similar to that used in Experiment 1 except that a different computer, a NOVA 800 , was used.

Stimuli. Stimuli were identical to those of Experiment 1, with the addition of the "quadrant-subtract" stimuli.

Procedure. Stimulus presentation and response procedures were identical to those of Experiment 1.

Design. The following changes were made in design. (1) Each subject participated in three separate sessions consisting of 3 blocks/session. The first session was considered practice. (2) A block consisted of 108 trials, resulting from 36 directions by 3 presentation methods. The circle response sheets were changed between blocks and during short breaks that occurred after the 36th and 72nd trials of each block. (3) Eight of the 16 subjects were placed in a "blocked" condition, while the other 8 were placed in a "mixed" condition. The mixed condition was similar to Experiment 1: Within each block, all 108 conditions were presented in random order. In the blocked condition, stimulus presentation method did not vary within a block. The three presentation methods were presented over the three blocks within a session in an order that was randomized for each subject and for each session.

\section{Results}

Data are presented collapsed over the second and third sessions. As in Experiment 1, the RTs are means of subject medians for each condition. As in Experiment 1, there was no significant interaction between quadrant and within-quadrant position; so the data are shown collapsed over these two variables in turn. Additionally, the blocked vs. mixed variable did not interact with anything, nor was there a significant main effect of blocked vs. mixed, so data are shown collapsed over blocked and mixed conditions.

Rotation data. Figure 3 shows RT as a function of rotation within a quadrant for the three presentation methods. The term "rotation within a quadrant" warrants a bit of explanation. For the numerical and quadrant-add conditions, rotation within a quadrant refers to exactly the same thing as has previously been called "position within a quadrant." However, for the

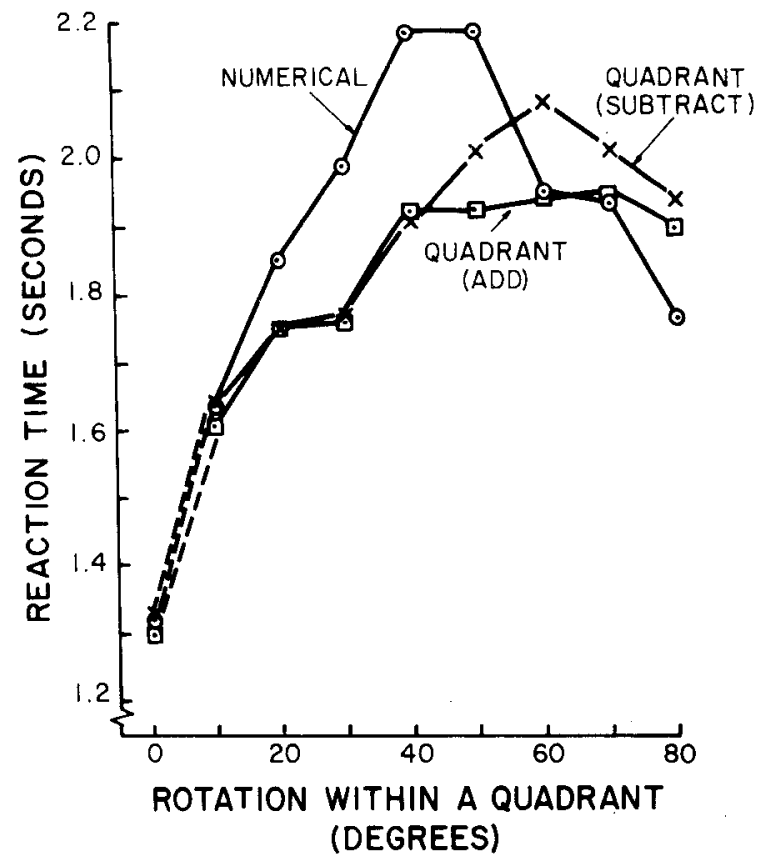

Figure 3. Experiment 2: Reaction time as a function of rotation within a quadrant.

quadrant-subtract condition, rotation within a quadrant refers to the number presented along with the cardinal direction. So, for example, the quadrant-subtract point for " 40 " is the average RT to "north minus 40 " (i.e., $320 \mathrm{deg}$ ), "east minus 40 " (50 deg), "south minus 40" (140 deg), and "west minus 40" (230 deg).

Several aspects of the data are noteworthy. First, the data from the numerical and quadrant-add conditions nicely replicate the data from Experiment 1 (Figure 1). Once again, the numerical curve was nomonotonic, reaching a peak at $40-50 \mathrm{deg}$, whereas the quadrant-add curve rose and then flattened out Second, the quadrant-add and quadrant-subtract curves were quite similar to each other, suggesting that the processes underlying the identification of, say, "north plus 20 " are much the same as those underlying the identification of "north minus 20. ."

There were highly significant statistical effects both of rotation $[\mathrm{F}(8,112)=55.5, \mathrm{MSe}=15.9]$ and of the Rotation by Presentation Method interaction $[\mathrm{F}(16,24)=11.9, \mathrm{MSe}=9.62]$. The main effect of presentation method was not significant $[F(2,29)=2.04$, $\mathrm{MSe}=50.47]$.

Additional statistical analyses were performed to isolate the nature of the various effects. First, an analysis was done comparing the quadrant-add and quadrantsubtract conditions only. This analysis resulted in no effect of presentation method or of the Method by Rotation interaction (both $F s<1$ ). A strong effect of rotation still emerged $[F(8,112)=41.0]$. Second, an analysis was done comparing the mean of the quadrant-add and quadrant-subtract conditions against the numerical condition. This analysis revealed no effect 
of presentation method $(F<1)$. However, there were strong effects of rotation $[F(8,112)=48.8]$ and of the Rotation by Method interaction $[F(8,112)=10.7]$. Finally, the data from Experiment 1 suggested no effect of rotation in the quadrant condition for rotations between 40 and $80 \mathrm{deg}$. To test this notion, an analysis was performed on the quadrant-plus and quadrant-minus conditions for rotation values of $40-80 \mathrm{deg}$ only. This analysis revealed no effect of method or interaction (both Fs $<1$ ). Additionally, there was no effect of rotation $[F(4,112)=1.27]$.

Quadrant data. Figure 4 shows RT for the four quadrants, collapsed over rotation within a quadrant. For the numerical and quadrant-add conditions, the representation is identical to that shown in Figure 2. Again, however, representation of the quadrant-subtract condition requires some clarification. Here, "quadrant" could refer to either the presented cardinal direction (e.g., "north minus X" would refer to the north quadrant) or to the quadrant in which the response is made (e.g., "north minus 0," "east minus X," $10 \leqslant X \leqslant 80$, would refer to the north quadrant). The representation is actually in terms of the former scheme. Thus, "north" responses would actually be made in the west quadrant, and so on.

Most of the remarks made about the rotation data (Figure 3) can be applied equally well to the quadrant data. The numerical and quadrant-add data provide a replication of Experiment 1; and there appears to be very little difference between the quadrant-add and quadrant-subtract conditions. There were highly significant effects of both quadrant $[\mathrm{F}(3,42)=54.5$, MSe $=14.16]$ and the Presentation Method by Quadrant interaction $[\mathrm{F}(6,84)=13.25, \mathrm{MSe}=15.58]$. An analysis comparing the quadrant-add and quadrant-subtract conditions revealed a significant quadrant effect $[F(3,42)=11.37]$ but no Presentation Mode by

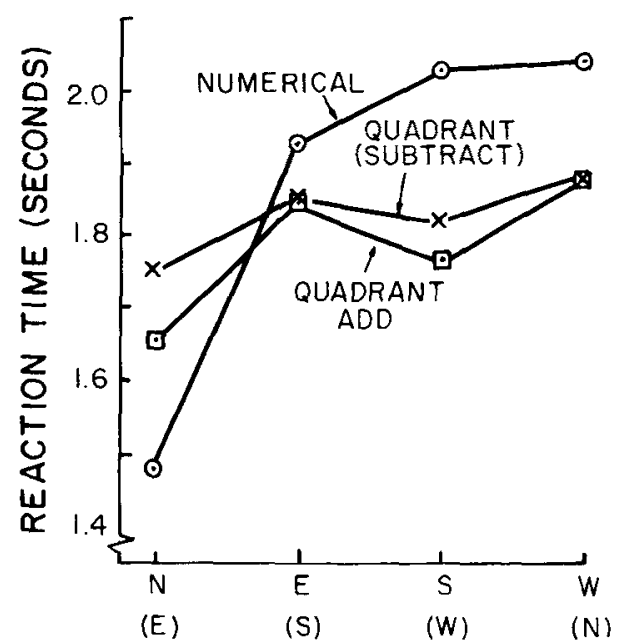

QUADRANT

Figure 4. Experiment 2: Reaction time for the four quadrants.
Quadrant interaction $(F<1)$. In contrast, an analysis pitting the mean of the two quadrant conditions against the numerical condition showed a significant interaction $[F(3,42)=19.07]$, as well as a significant quadrant effect $[F(3,42)=55.91]$. In short, the two quadrant conditions were once again behaving quite similarly to each other and quite differently from the numerical condition.

\section{GENERAL DISCUSSION}

The results of both experiments have implications for (1) normal direction comprehension, (2) the degree to which normal direction comprehension can be modified, and (3) representation of directional information.

\section{Normal Comprehension of Direction}

The numerical mode represents a typical means of transmitting directional information. The rotation results of both experiments (Figures 1 and 3) suggest that comprehension of a particular direction may be comprised of two steps: First, computing the nearest cardinal direction, and second, rotation from the cardinal to the desired direction.

Two separate aspects of the data suggest that rotation can be carried out equally well in a clockwise or a counterclockwise direction. First, the rotation curves from the numerical conditions are remarkably symmetrical around $45 \mathrm{deg}$. Second, the near identity of the quadrant-add and quadrant-subtract rotation curves indicate that clockwise and counterclockwise rotations involve quite similar processes.

\section{Quadrant-Mode Comprehension}

The process by which directions are understood, when presented in the quadrant mode, is rather more mysterious. Consider first the rotation data (Figures 1 and 3). For rotations between 0 and $40 \mathrm{deg}$, it appears that the process of finding a direction within a quadrant may be similar for the quadrant and numerical modes. Why, though, are the quadrant-mode curves flat between 40 and $80 \mathrm{deg}$ ? The most straightforward hypothesis is that the flat curves are the averaged results of two separate strategies. That is, perhaps some subjects always rotate in the "approved" direction (clockwise in the quadrant-add mode; counterclockwise in the quadrantsubtract mode), whereas other subjects still compute and start from the nearest cardinal direction, even in the quadrant mode. Evidence for this hypothesis was sought in the following way. Consider the 16 subjects in Experiment 2. For each subject, two 5-point curves were plotted: the rotation curves for rotations of 40-80 deg for the quadrant-add and quadrant-subtract . conditions. These curves are shown in Figure 5. Now, according to the "two-strategy averaging" hypothesis, each of these individual curves should be monotonically 

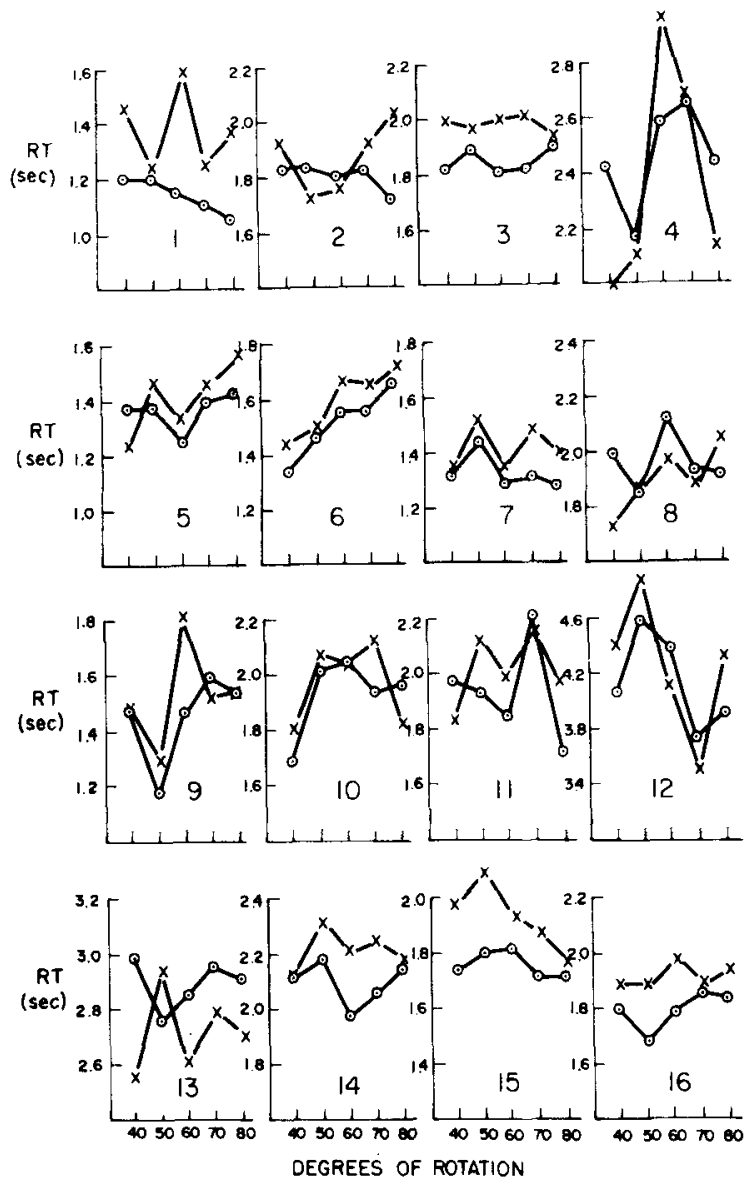

Figure 5. Experiment 2: Individual subject data. Functions relate reaction time to rotation within a quadrant for rotations of 40-80 deg. The two curves in each graph correspond to the quadrant-add condition (Os) and quadrant-subtract condition (Xs). Numbers in each graph are subject numbers.

increasing or decreasing. By and large, this was not so, as is quite readily apparent from inspection of the curves. Departures from monotonicity are reflected in the number of reversals that exist in a given curve: Zero reversals would reflect perfect monotonicity, and a maximum of three reversals could potentially be made in a 5-point curve. Table 1 shows the distribution of numbers of reversals over the 32 curves. Only two of the curves are perfectly monotonic, and the modal number of reversals is three. A rough statistical test of monotonicity was executed as follows. A null hypothesis may be constructed stating that the five points constituting any given curve consist of five values randomly drawn from a single distribution. This null hypothesis yields the expected reversal frequency distribution shown in the second column of Table 1. These two distributions did not differ from each other $\left[\chi^{2}(2)=.30\right]$.

Thus, it seems unlikely that the populations underlying the curves in Figure 5 are populations of monotonic curves. Does this mean that the processes underlying responses to rotations between 40 and $80 \mathrm{deg}$ are processes that give rise to flat curves? A second analysis of the Figure 5 curves suggests that this is not the case either. This analysis involved simply correlating the two curves for each of the 16 subjects. Thirteen of the 16 correlations are positive, as shown in Table 2 . If the curves of Figure 5 were noisy samples drawn from an underlying population of flat curves, then each pair of curves would tend to be uncorrelated. It therefore appears that there is a variety of idiosyncratic strategies as opposed to just one or two. A given subject appears to apply a given strategy to both quadrant-add and quadrant-subtract responses; and the result of averaging the various strategies is a curve that is more or less flat.

\section{What is "Rotated"?}

The foregoing discussion has been concerned with the strong influence on RT of rotation within a quadrant. Referring to this influence as the "rotation effect," an obvious question is: What psychological processes are responsible for it? It is tempting to draw an analogy between this rotation effect and the "mental rotation" effects reported by Shepard and his co-workers (e.g., Cooper \& Shepard, 1973; Shepard \& Metzler, 1971). However, such an analogy would probably be premature at this point. Shepard's experiments always involve the presentation of a concrete visual stimulus such as a letter or a picture, and the stimulus display is always such that a physical rotation of the stimulus would be sufficient to perform the experimental task. It is therefore quite natural to model the processes involved by assuming a secondorder isomorphism. That is, an internal representation of the visual stimulus is assumed to be formed and this

Table 1

Observed and Expected Distributions of Number of Reversals for the 32 Curves Shown in Figure 5

\begin{tabular}{ccc}
$\begin{array}{c}\text { Number of } \\
\text { Reversals }\end{array}$ & Observed & Expected \\
\hline 0 & 2 & .53 \\
1 & 7 & 7.47 \\
2 & 14 & 15.47 \\
3 & 9 & 8.53 \\
\hline
\end{tabular}

Note $-\chi^{2}(2)=.30 ;$ frequencies of 0 and 1 are collepsed.

Table 2

Correlations of Quadrant-Add and Quadrant-Subtract Conditions for Rotations of $\mathbf{4 0 - 8 0} \mathrm{deg}$

\begin{tabular}{cccc}
\hline Subject & $\mathrm{r}$ & Subject & $\mathrm{I}$ \\
\hline 1 & .27 & 9 & .57 \\
2 & -.74 & 10 & .64 \\
3 & -.62 & 11 & .45 \\
4 & .67 & 12 & .86 \\
5 & .54 & 13 & -.66 \\
6 & .86 & 14 & .71 \\
7 & .65 & 15 & .65 \\
8 & .06 & 16 & .09 \\
\hline
\end{tabular}


representation then undergoes an operation that is akin to what is involved during the perception of a physical rotation. A physical rotation may thus be viewed as a model of internal processes and ensuing predictions fall out quite easily.

However, the present experimental paradigm is quite different in the sense that there is no physical stimulus that could be rotated in order to answer the question at hand. It is possible, of course, that the same mechanisms that underlie the rotation effects in the Shepard paradigms also underlie the rotation effect in the present experiments. One could, for example, speculate that the subject rotates an internal representation of him/herself, or perhaps an internal representation of the to-be-drawn line. But until such a class of hypotheses is experimentally tested, it seems prudent to keep the term "rotation" at a metaphorical level.

\section{Quadrant Effects}

Whatever may cause it, the rotation effect is assumed to reflect processes involved in arriving at a desired direction within a particular quadrant. The data shown in Figures 2 and 4 bear on the issue of how a particular quadrant is arrived at to begin with.

When the numerical mode is used, there is a monotonic increase in RT clockwise around the four quadrants. There are several possible reasons for this effect. First, one might argue that the effect stems from motor differences; that is, it might, for some reason, be increasingly difficult simply to draw a line around the four quadrants. This possibility is unlikely, for reasons to be described below. Second, it has been noted above that subjects appear to compute the nearest cardinal direction (i.e., the nearest multiple of $90 \mathrm{deg}$ ) when presented with numerical-mode information. Possibly, computing the nearest multiple of 90 for some number is more difficult, the higher is the number. A theory of the processes necessary to compute multiples of 90 is beyond the scope of this paper. But such a hypothesis seems reasonable on intuitive grounds.

Consider now quadrant effects for information presented in the quadrant mode. On an a priori basis, it seemed entirely reasonable that there would be no quadrant effects for this presentation method. That is, there seemed to be no reason to expect that, say, "north plus 40 " would differ from "east plus 40 ." Contrary to this expectation, rather substantial and systematic differences appeared: The responses initiated by north and south were faster than the responses initiated by east and west. (Of passing interest is the fact that naive subjects showed a further advantage of north over south, whereas pilots showed no such advantage.)

What is responsible for these quadrant effects? Two reasons permit rejection of the possibility that they are motor effects. First, the quadrant-plus and quadrant-minus curves are similar in form, even though they involve responding in different physical quadrants.
Second, the patterns of the quadrant-mode and numerical-mode curves are so different that it is difficult to imagine a common component of physical quadrant effects.

Rather, it appears that subjects are inherently faster in comprehending where north and south are as opposed to where east and west are. Such a conclusion dovetails quite nicely with previous findings that left-right discriminations are more difficult than are up-down discriminations, both in children (Rudel \& Teuber, 1963) and in adults (Just \& Carpenter, 1975; Maki, Maki, \& Marsh, 1977). The Maki et al. study was the most complete in demonstrating this differential difficulty, indicating that left-right discriminations took longer than up-down discriminations (1) for both memorized familiar stimuli (e.g., U.S. states) and for perceptually presented artificial stimuli; (2) for both locational information (e.g., "left of," "above," etc.) and orientational information ("vertically oriented" vs. "horizontally oriented"); and (3) when either up, down, left, and right or north, south, east, and west were used to denote relative position. No universal explanation has been pinned down for these effects; however, it has been speculated that they may reflect the fact that the human brain has a left-right but not an up-down symmetry (e.g., Corballis \& Beale, 1970; 1976).

\section{REFERENCES}

Corballis, M. C., \& Beale, I. L. Bilateral symmetry and behavior. Psychological Review, 1970, 77, 451-464.

Corballis, M. C., \& BeAle, I. L. The psychology of left and right. Hillsdale, N.J: Lawrence Erlbaum, 1976.

COOPER, L. A., \& SHEPard, R. N. Chronometric studies of the rotation of mental images. In W. Chase (Ed.), Visual information processing. New York: Academic Press, 1973.

Downs, R. M., \& StEA, D. Image and environment. Chicago: Aldine, 1973.

Just, M. A., \& CARpenter, P. A. The semantics of locative information in pictures and mental images. British Journal of Psychology, 1975, 66, 427-441.

Lrnch, K. The image of the city. Cambridge: MIT Press, 1960.

Maki, R. H., Maki, W. S., \& Marsh, L. G. Processing locational and orientational information. Memory \& Cognition, 1977, 5, 602-612.

Rudel, R., \& TEUBER, H. L. Discrimination of direction of line in children. Journal of Comparative and Physiological Psychology, 1963, 56, 892-898.

Shepard, R. N., \& Metzler, J. Mental rotation of threedimensional objects. Science, 1971, 171, 701-703.

Tolman, E. C. Cognitive maps in rats and men. Psychological Review, 1948, 55, 189-208.

\section{NOTES}

1. In this paper, the claim of a significant effect indicates an $\alpha$ value of less than .05 and the claim of a nonsignificant effect indicates an $\alpha$ value of greater than .10 .

2. Mean square errors are presented as squared deciseconds.

(Received for publication February 13, 1978; accepted April 4, 1978.) 